\title{
Soft Computing based Model for Identification of Pseudoknots in RNA Sequence using Learning Grammar
}

\author{
Ankita Jiwan, Shailendra Singh \\ Department of Computer Science and Engineering, \\ PEC University of Technology, Chandigarh
}

\begin{abstract}
RNA structure prediction is one of the major topics in bioinformatics. Among the various RNA structures, pseudoknots are the most complex and unique structure. Various methods have been used for modeling RNA pseudoknotted secondary structure. In this paper a new model for prediction of RNA pseudoknot structure has been proposed. In this model, features of two existing techniques, i.e. neural network and grammar are combined. The advantage of grammar, identification based on rules is combined with the strength of a neural network to learn. An Elman neural network is used to learn the context free grammar that represents a pseudoknot. This Learning grammar network further identifies if the RNA sequence contains pseudoknot or not. Learning grammar helps in reducing the drawbacks of both neural network and grammar thus increasing the overall power of identifying sequences with pseudoknots.
\end{abstract}

\section{Highlights}

- Pseudoknots are the most complex and unique structure. It is very difficult for an algorithm to identify all the classes of pseudoknots at once.

- In the proposed Learning Grammar Model for Pseudoknot Identification features of existing technology neural network and grammar are combined.

- In this model Elman neural network is used. Elman neural network tries to learn context free grammar that represents a pseudoknot. After learning the neural network can classify RNA sequence into sequences with or without pseudoknots.

- For sequences with pseudoknots the model could detect $80.34 \%$ of the sequences. It could detect all of the sequences without pseudoknots correctly.

- Combining neural network with grammar helps in reducing the drawbacks of both the technologies and increasing the overall power of identifying sequences with pseudoknots

Keywords-- Minimum Free Energy, Pseudoknots, Soft Computing, Elman Neural Network, Grammar, Context Free Grammar

\section{INTRODUCTION}

Bioinformatics is an applied science where mathematical and computational theories and technologies are used in order to process, relate and derive predictions and inferences from data obtained in molecular biology. Bioinformatics' goal is to understand and analyze the information control and flow within different organisms. There is a synergic interaction between computer science, mathematics and biology, each with its own richness and limitations [1].

RNA structure prediction has been a major topic of research and especially predicting structure of the non coding RNA. An ncRNA folds into a characteristic structure depending upon the interactions complementary base pairs. Out of the ncRNA secondary structures pseudoknots are the most distinct structure. The paper gives a description of pseudoknot prediction techniques and is divided into the following sections. Firstly it includes biological basics of RNA pseudoknot, definition and classes of pseudoknot. Followed by details on popular pseudoknot prediction techniques i.e. dynamic programming, grammatical approach and soft computing. Finally discussing learning grammar model for predicting the pseudoknot in RNA sequence along with the research techniques combined in the new model.

\section{BIOLOGICAL BASICS OF RNA PSEUDOKNOT}

DNA molecules are composed of nucleotides which carry the biological information [5]. RNA a singlestrand molecule is made up of nucleotides that are adenine (A), cytosine (C), uracil (U), and guanine (G) which then bounded with sugar-phosphate backbone. Among these four nucleotides cytosine (C) and uracil (U) are pyrimidines and adenine (A) and guanine (G) are puriens. To form a stable RNA structure puriens bond with pyrimidines which means adenine (A) bonds with uracil (U) and cytosine $(\mathrm{C})$ bonds with guanine $(\mathrm{G})$.

Single-stranded RNA sequence folds over to form secondary structures like hairpin loops, internal loops, double helix, bulge loops (called orthodox RNA structure), pseudpoknots (called non-orthodox RNA structure) etc reducing their energy to form a stable structure[4].

A non-coding RNA (ncRNA) folds to form characteristic structure depending upon the interactions complementary base pairs. Out of the ncRNA secondary 
structures pseudoknots are the most typical. The pseudoknots play a vital role in translation, viral genome structure, ribosome active site and many others [6] [3].

\section{Definition and classes of pseudoknots}

When bases pair between nucleotides loops (hairpin or internal) and bases outside the enclosing loop, interact they form a pseudoknot. This structure often contains coaxial helices. It can be a very stable tertiary interaction [9].

Let $\mathrm{S}=\mathrm{s}_{1} \mathrm{~s}_{2} \ldots \mathrm{s}_{\mathrm{m}}$ be a RNA sequence and $\mathrm{Q}$ be the secondary structure of $\mathrm{S}$. Q is represented as a set of base pairs $\left(\mathrm{s}_{\mathrm{i}}, \mathrm{s}_{\mathrm{j}}\right), 1 \leq \mathrm{i}<\mathrm{j} \leq \mathrm{m}$.

Let $\mathrm{Q}_{\mathrm{x}, \mathrm{y}} \subseteq \mathrm{Q}$ be the set of base pairs in the subsequence $\mathrm{s}_{\mathrm{x}} \mathrm{s}_{\mathrm{x}+1} \ldots \mathrm{s}_{\mathrm{y}}, 1 \leq \mathrm{x}<\mathrm{y} \leq \mathrm{m}$.

$$
\mathrm{Q}_{\mathrm{x}, \mathrm{y}}=\left\{\left(\mathrm{s}_{\mathrm{i}}, \mathrm{s}_{\mathrm{j}}\right) \in \mathrm{Q} \mid \mathrm{x} \leq \mathrm{i}<\mathrm{j} \leq \mathrm{y}\right\} .
$$

$\mathrm{Q}_{\mathrm{x}, \mathrm{y}}$ is a regular structure (as in figure 1(a)) if there does not exist two pairs

$(\mathrm{i}, \mathrm{j}),(\mathrm{k}, \mathrm{l}) \in \mathrm{Q}_{\mathrm{x}, \mathrm{y}}$ such that

$$
\mathrm{i}<\mathrm{k}<\mathrm{j}<1 \text { or } \mathrm{k}<\mathrm{i}<\mathrm{l}<\mathrm{j} .
$$

An empty set is considered as a regular structure.

$\mathrm{Q}_{\mathrm{x}, \mathrm{y}}$ is a simple pseudoknot (as in figure 1(c)) if $\exists \mathrm{x}<$ $\mathrm{x}_{1}, \mathrm{x}_{2}<\mathrm{y}\left(\mathrm{x}_{1}, \mathrm{x}_{2}\right.$ are referred as pivot points) such that

i) each (i, j) $\in Q_{x, y}$ satisfies either $x \leq i<x 1 \leq j<x 2$ or $\mathrm{x} 1 \leq \mathrm{i}<\mathrm{x} 2 \leq \mathrm{j} \leq \mathrm{y}$; and,

ii) $Q_{L}$ and $Q_{R}$ are both regular where

$$
\begin{aligned}
& \mathrm{Q}_{\mathrm{L}}=\left\{(\mathrm{i}, \mathrm{j}) \in \mathrm{Q}_{\mathrm{x}, \mathrm{y}} \mid \mathrm{x} \leq \mathrm{i}<\mathrm{x} 1 \leq \mathrm{j}<\mathrm{x} 2\right\} \text { and } \\
& \mathrm{Q}_{\mathrm{R}}=\left\{(\mathrm{i}, \mathrm{j}) \in \mathrm{Q}_{\mathrm{x}, \mathrm{y}} \mid \mathrm{x} 1 \leq \mathrm{i}<\mathrm{x} 2 \leq \mathrm{j} \leq \mathrm{y}\right\} .
\end{aligned}
$$

$\mathrm{Q}$ is a recursive simple pseudoknot (as in figure 1(d)) if $\exists 1 \leq \mathrm{x}_{1}<\mathrm{y}_{1}<\ldots<\mathrm{x}_{\mathrm{s}}<\mathrm{y}_{\mathrm{s}} \leq \mathrm{m}$ such that

i) $\mathrm{Q}_{\mathrm{xi}, \mathrm{yi}}$, for $1 \leq \mathrm{i} \leq \mathrm{s}$, is a recursive simple pseudoknot, simple pseudoknot, or a regular structure and,

ii) $\left(\mathrm{Q}-\mathrm{U}_{1 \leq \mathrm{i} \leq \mathrm{s}} \mathrm{Q}_{\mathrm{xi}, \mathrm{yi}}\right)$ is a regular or a simple pseudoknot structure.

Embedded simple pseudoknot structure is a subset of recursive simple pseudoknot. It is defined as follows: [11].

$\mathrm{Q}$ is an embedded simple pseudoknot structure if $\exists 1 \leq$ $\mathrm{x}_{1}<\mathrm{y}_{1}<\ldots<\mathrm{x}_{\mathrm{s}}<\mathrm{y}_{\mathrm{s}} \leq \mathrm{m}$ such that

i) $\mathrm{Q}_{\mathrm{xi}, \mathrm{yi}}$, for $1 \leq \mathrm{i} \leq \mathrm{s}$, is a simple pseudoknot structure and,

ii) $\left(\mathrm{Q}-\mathrm{U}_{1 \leq \mathrm{i} \leq \mathrm{s}} \mathrm{Q}_{\mathrm{xi}, \mathrm{yi}}\right)$ is a regular structure.

There is another restricted class of recursive simple pseudoknot which is also found in existing ncRNA families. This class is referred as 2-level recursive simple pseudoknot with regular recursive regions. Q is a 2-level recursive simple pseudoknot with regular recursive regions if $\exists 1 \leq \mathrm{x}_{1}<\mathrm{y}_{1}<\ldots<\mathrm{x}_{\mathrm{s}}<\mathrm{y}_{\mathrm{s}} \leq \mathrm{m}$ such that

i) $\mathrm{Q}_{\mathrm{xi}, \mathrm{yi}}$, for $1 \leq \mathrm{i} \leq \mathrm{s}$, is regular and,

ii) $\left(\mathrm{Q}-\mathrm{U}_{1 \leq \mathrm{i} \leq \mathrm{s}} \mathrm{Q}_{\mathrm{xi}, \mathrm{yi}}\right)$ is a simple pseudoknot structure.

Simple pseudoknot structure is a subset of both the 2level recursive simple pseudoknot with regular recursive regions and the embedded simple pseudoknot structure. The latter two classes of pseudoknots are subsets of recursive simple pseudoknot structure [7].

\section{RELATED RESEARCH WORK}

RNA pseudoknots have drawn considerable attention of researchers because they give 3-D structure to the molecule, a structure that will determine in most cases its particular biological function. Even though determining the molecular structure is of vital importance. It is also particularly hard and expensive to obtain structural data from RNA spectrometry and crystallography [1].

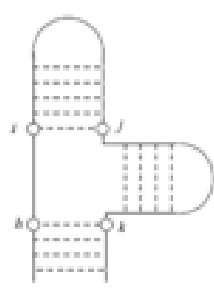

(a)

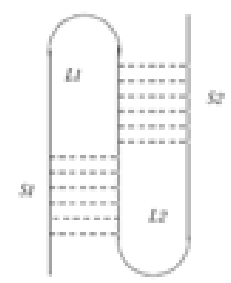

(c)

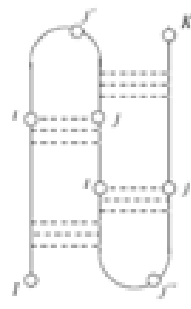

(b)

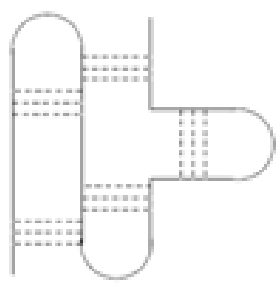

(d)
Figure 1 Examples of RNA secondary structure:

(a) Simple (non-pseudoknotted) structure; (b) Pseudoknot; (c) H-pseudoknot (simple pseudoknot); (d) Recursive pseudoknot.

Among the many exponential possibilities of secondary structure for a given RNA sequence under fixed environmental conditions, the most stable structure is with the minimum free energy. Free energy can be calculated by summation of energies (entropy and thermodynamic) related to secondary structure's components. Estimating this energy becomes an NP-hard problem [1]. There is a tradeoff between the algorithm's complexities and the generalization of RNA structures classes [17]. Many techniques like dynamic programming, grammatical approach, soft computing techniques and more exist to predict the structure of RNA pseudoknots [14]. 


\subsection{RNA Pseudoknot Structure Prediction Techniques}

The following techniques exist to predict the structure of RNA pseudoknots:

\subsubsection{Dynamic programming}

Dynamic programming is a very well known and usefull method for solving complex problems by breaking them down into simpler subproblems. Using this concept of dividing into subproblems and the concept of Minimum Free Energy, many algorithms were developed. Some popular algorithms among the many dynamic programming algorithms were proposed by Rivas and Eddy (R\&E) [10], Lyngso and Pedersen (L\&P) [12], and Dirks and Pierce (D\&P) [8].

Dirks and Pierce (D\&P) algorithm is more general than the others, this can be said because calculations can be made using the partition function as well as the Minimum Free Energy secondary structure [2]. It has time complexity $\Theta\left(\mathrm{n}^{5}\right)$ and space complexity is $\Theta\left(\mathrm{n}^{4}\right)$. Lyngso and Pedersen (L\&P) algorithm is for a restricted class, which includes structure of the form $\mathrm{x}_{1} \mathrm{x}_{2} \mathrm{x}_{1}{ }^{\prime} \mathrm{x}_{2}$ ' where both $\mathrm{x}_{1} \mathrm{x}_{1}{ }^{\prime}$ and $\mathrm{x}_{2} \mathrm{x}_{2}{ }^{\prime}$ are pseudoknot free structures. The time complexity and space complexity is $\Theta\left(n^{6}\right)$ and $\Theta\left(n^{4}\right)$ respectively. Rivas and Eddy (R\&E) is a complete model with possibility to calculate the free energy and find parameters of pseudo-knotted secondary structures [2]. However, the complexity of algorithm makes it infeasible for molecules of larger length. Its worst case time complexity is $\Theta\left(\mathrm{n}^{6}\right)$ and $\Theta\left(\mathrm{n}^{4}\right)$ for space restricting the sequence to a maximum length of around 150 nucleotides [12].

\subsubsection{Grammatical Approach}

In grammatical approach, secondary structure prediction is viewed as a parsing problem. Several grammars have been used to predict the pseudoknotted RNA structure. A comparison of grammars show significant attention is paid on context-free grammar to predict the orthodox RNA structure which took $\Theta\left(\mathrm{n}^{3}\right)$ time where $\mathrm{n}$ was the length of the input sequence. Many variations of $\mathrm{CFG}$ like Scholastic Context Free Grammar (SCFG), Multiple Context Free Grammar (MCFG), and Scholastic Multiple Context Free Grammar (SMCFG) can better represent pseudoknots.

Many attempts have been made at modeling RNA structure by formal language. Few grammars have been proposed to represent pseudoknots. The pioneer define two subclasses of tree adjoining grammar (TAG) called simple linear tags (SL-TAG) and extended simple linear tags (ELS-TAG), and argue that ELS-TAG is appropriate for representing RNA secondary structure including pseudoknots [23]. SL-TAG cannot be used to represent pseudoknots because of its limited representative power. Whereas ELS-TAG is the minimum grammar that can define a pseudoknot structure grammatically.

Later grammars like SS-TAG (a sub class of TAG) was introduced, it is more general as compared to ELS-TAG.
Advancement in Context Free Grammar similar to Scholastic Context Free Grammar (SCFG) [18] [13], Multiple Context Free Grammar (MCFG) can better define a pseudoknot.

It is difficult to answer which class of grammar is the minimum to represent pseudoknots as no exact definition of pseudoknots in a biological or geometrical sense is available. The generative power of ELS-TAG, SS-TAG and MCFG was found to be same of $\Theta\left(n^{5}\right)$ [24].

A new grammar, Multiple Context Free Grammar along with probability of the rule called the Scholastic Multiple Context Free Grammar (SMCFG) is recently introduced for predicting pseudoknotted RNA structures the [15] [1]. SCFG has the generative power and accuracy comparable to dynamic programming method. One of the important parameter used in the analysis is Fmeasure. F-measure is weighted harmonic mean of precision and recall. The F-measure is also known as balanced F-score. It average F-measure is $65 \%$.

Only grammatical approach is not sufficient i.e. we need more technology to be included into grammatical approach to increase its performance.

\subsection{3. $\quad$ Soft computing}

Soft computing is a group of methodologies that work together and provides capabilities for handling real life ambiguous and uncertain situations. The aim of soft computing is to exploit the tolerance for approximate reasoning, imprecision, uncertainty and partial truth in order to achieve tractability, robustness, low solution cost, and close resemblance with human like decisionmaking [2].

The previously discussed techniques have high time and space complexities, which lead the use of soft computing techniques in the prediction of RNA pseudoknots [19]. Among the many soft computing techniques that could be used for pseudoknot prediction like Artificial Neural Network, Genetic Algorithms, Fuzzy Neural Network, Swarm Intelligence to achieve near optimal results in less computational time and memory requirements. Out of these soft computing techniques the neural network is very useful and versatile.

Artificial Neural Network (ANN) is composed of interconnecting artificial neurons (programming units that mimic the properties of biological neurons to learn). Artificial neural networks aim in solving artificial intelligence problems by gaining knowledge through learning. Artificial Neural Network based algorithm to predict the RNA secondary structure use maximizing base pairs. They find the most stable structure, the structure with minimum energy. More the number of base pairs found more stable the secondary RNA structure will be. Some ANN following this paradigm are described in [22] [16] [20]. The Back Propagation neural network (BPN) is widely used neural network. The learning of BPN is supervised type. The error is calculated in the last layer and feedback to previous layers to modify the weights. A simple recurrent network 
is used in [20] to classify and predict the best secondary structure. The neural network like Hopfield which acts like a content addressable memory unit are used in [22] [16]. ANN can predict the complex pseudoknots with the same ease as the orthodox structures. The complexity of designing and learning may increase but the predictions are simple with minimum resource requirements.

\section{PROPOSED MODEL}

In this model the advantage of grammar, identification based on rules is combined with the strength of a neural network to learn. These technologies combine to form the Learning Grammar Model for pseudoknot identification. The technologies are explained below:

\section{Exiting Technologies Used in the Learning Grammar Model}

\subsection{Context Free Grammar for RNA Pseudoknot}

The simplest grammar that can represent a pseudoknot is context free grammar, various types of non terminal symbols are needed to model the different known structures.

The Non-terminals of the grammar and their semantic are:

- P: the paired columns in Watson-Crick bridges (bonds A-U C-G) are described by a non terminal that emits a base pairing.

- L: The non paired columns are described by a non terminal that emits to the left (direction 5'_3') whenever possible; i.e., when no possible ambiguous sequences may arise.

- R: non terminal that emits to the right (direction 3' 5'). Case that can occasionally happen in protuberances between stems and loops in the right part of the structure (strand 3'). It is used when ambiguous sequences emerge when $\mathrm{L}$ is used.

- B: Bifurcation non terminal used to split several stems or loops with various branches arising from it.

- S: Beginning non terminal that acts as immediate son to a bifurcation's derivation or a sequence start.

- E: Ending non terminal that finishes the derivation of sequences.

- D: Suppression non terminal i.e. used to describe a production that does not emit terminal symbols and does not describe one of the previous cases.

Each one of the non terminals has, by its stochastic characteristic, a probability $\mathrm{e}_{\mathrm{v}}(\mathrm{a}, \mathrm{b})$ of emitting one or two pairs of bases. Here $\mathrm{v}$ is the non terminal and $a, b \in\{A, G, C, U\}$. The symbol $t_{v}(Y)$ represents the probability to go from state $\mathrm{v}$ to state $\mathrm{Y}$ [18].

$\begin{array}{ll}\mathrm{P} \rightarrow \mathrm{aYb} & \begin{array}{l}\text { Pair derivation (16 possible } \\ \text { emitting types) } \\ \text { Left derivation (4 possible } \\ \text { emitted symbols) } \\ \mathrm{R} \rightarrow \mathrm{aY}\end{array} \\ \begin{array}{l}\text { Right derivation (4 possible } \\ \text { emitted symbols) }\end{array} \\ \mathrm{B} \rightarrow \mathrm{SS} & \text { Bifurcation } \\ \mathrm{D} \rightarrow \mathrm{Y} & \text { No symbol derivation } \\ \mathrm{S} \rightarrow \mathrm{Y} & \text { Start } \\ \mathrm{E} \rightarrow \epsilon & \text { End }\end{array}$

4.2. Neural Network to Learn Grammar (ELMAN Network)

Memory is needed to learn temporal information. There are two basic ways to build memory into the neural networks [27]. The first one is to introduce time delays in the network and to adjust their parameters during the learning phase. The second way is using positive feedback, i.e. making the network recurrent. To characterize memories in different architectures, two dimensions, depth and resolution has been proposed. Roughly, depth refers to how far into the past the memory stores information relative to the memory size, and resolution how accurately information concerning the individual $\mathrm{y}(\mathrm{n})=\mathrm{x}^{\wedge}(\mathrm{n})$ elements of the input sequence is preserved, where $\mathrm{n}$ is number of elements [26]. In time series prediction with neural networks the main problems have been deciding prediction order and the structure of the network [25].

In the case of grammars, it may be verified that recurrent neural networks have an inherent ability to simulate finite state automata [30], from which grammars of regular languages are inferred. The behavior of a recurrent neural net (RNN), a dynamical system can be used to construct finite state automaton [30]. However, regarding the natural language processing, it must be noted that grammars of natural languages cannot be completely represented by finite state models, due to their hierarchical structures [31]. Nevertheless, it has been shown that recurrent networks have the representational power required for hierarchical solutions [29], and therefore, a type of RNN for natural language processing, called Elman network can be used [28]

The Elman network takes positive feedback to construct memory in the network as shown in Figure 2 . The network has input, hidden and output layers. 


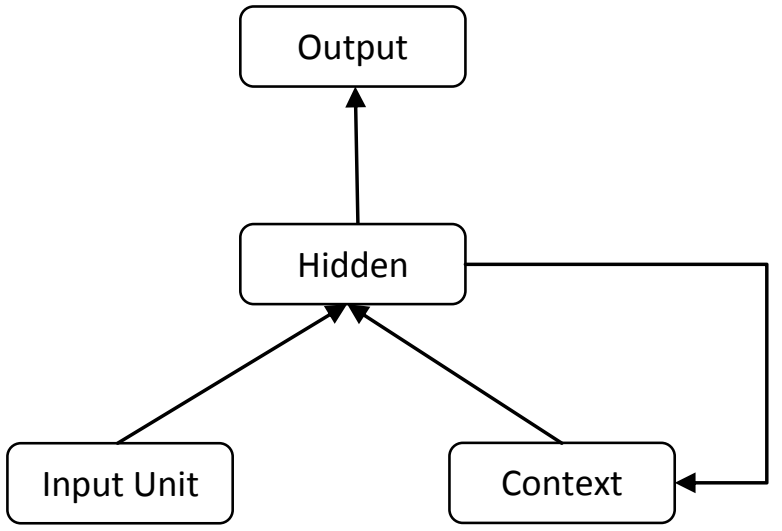

Figure 2 Elman Neural Network

Special units called context units save previous output values of hidden layer neurons. Context unit values are then fed back fully connected to hidden layer neurons and thus they serve as additional inputs to the network. Networks output layer values are not fed back to network.

The Elman network has a high depth, low resolution memory, since the context units keep exponentially decreasing in trace of past hidden neuron output values. The memory in the network has no rigid limit, and the fact that the information concerning previous data is preserved with better resolution than more distant data in the past.

\section{LEARNING GRAMMAR MODEL FOR PSEUDOKNOT IDENTIFICATION}

In this model, features of existing technology neural network with grammar as discussed above are combined. The neural network learns grammar to identify pseudoknot in RNA sequence. The model is shown in figure 3 and its 3 steps are explained as follows:

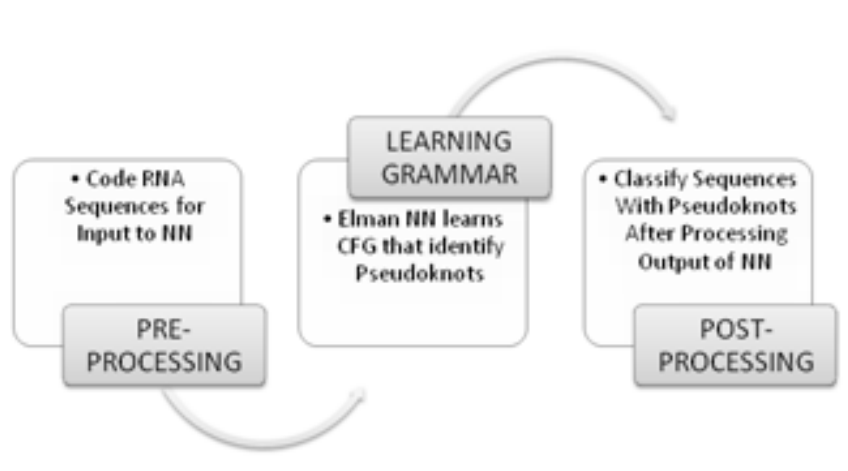

Figure 3 Learning Grammar Model for Pseudoknot Identification

\section{i. $\quad$ Pre-Processing}

The selection of an appropriate input encoding method is probably one of the most significant factors determining the performance of the final system, because the encoding determines what information is presented to the NNs. An idea1 encoding scheme should extract maximal information from the sequence, and satisfy the basic coding assumption so that similar sequences are represented by close vectors. The design for the input sequence encoding method is application dependent, and affected by many other considerations. Among the two encoding techniques, i.e. direct or indirect encoding any one can be used [21].

The RNA sequence is coded in binary format in order to be fed to the neural network. The four alphabets of an RNA sequence A, G, C, T are coded in a matrix of $m X n$ where $n$ is the length of the sequence and $\mathrm{m}$ is the number of bits used to code each alphabet of the sequence. There are two popular encoding schemes used for RNA sequences. One of the encoding schemes is BIN4 in which 1000 (A), $0100(\mathrm{U}), 0010(\mathrm{G})$, and $0001(\mathrm{C})$. In the other encoding scheme the alphabets are coded as 1101 (A), $0100(\mathrm{U}), 1000(\mathrm{G}), 1110(\mathrm{C})$.

\section{ii. Learning Grammar}

Elman Neural Network can be used to learn the context free grammar of pseudoknot. The number of neurons in the input layer and output layer is equal to $\mathrm{m}$ (encoding scheme of the sequence). The number of neurons in the context unit can vary according to the level of generalization.

The Elman network will take an alphabet of the RNA sequence and predict the next alphabet in the sequence according to the grammar representing pseudoknots. The neural network can thus learn all classes of pseudoknots at once using only the context free grammar.

\section{iii. Post-Processing}

After the neural network has learnt the grammar the output of the neural network can be used to identify sequences with pseudoknots. The network when fed with the input symbol predicts the next symbol in the sequence according to the grammatical rules learnt. If the alphabets predicted by the neural network is same as the alphabets in the actual sequence then the sequence has a pseudoknot otherwise the sequence does not have a pseudoknot. This criterion can be used to identify sequences with and without pseudoknots.

Pseudoknots are the most complicated secondary structure among all RNA secondary structures. It is very difficult for an algorithm to identify all the classes of pseudoknots at once. Hence there is a tradeoff between the algorithm's complexities and the generalization of RNA structures classes [2]. The complexity of grammatical approaches is of the $\mathrm{O}\left(\mathrm{n}^{5}\right)$ making it very time consuming whereas neural network reduces the time complexity but the time required in designing a 
neural network is higher than other techniques. Combining neural network with grammar helps in reducing the drawbacks of both the technologies and increasing the overall power of identifying sequences with pseudoknots. There is a tradeoff between time necessary for constructing a system to identify pseudoknots and time taken in executing and producing the required results.

\section{RESULTS}

RNA pseudoknot sequences from the PseudoBase++ (http://pseudobaseplusplus.utep.edu/home) were used to test the Learning Grammar Model for Pseudoknot Identification. The developed model is tested with 300 sequences containing pseudoknots. The model could detect $80.34 \%$ of the sequences correctly as sequences with pseudoknots. Further testing the model with nonpseudoknots sequences, it could detect all of the sequences correctly i.e. sequences without pseudoknots.

\section{CONCLUSION}

Pseudoknots are complex and unique secondary structure among all RNA secondary structures. All the classes of pseudoknots i.e. simple pseudoknot, H-pseudoknot, recursive pseudoknot cannot be identified by an algorithm at once. There always exists a tradeoff between the algorithm's complexities and the generalization of class of pseudoknot it can identify. The learning grammar model tries to overcome this problem. Elman neural network used in the model tries to learn context free grammar that represents a pseudoknot. After learning this neural network can now classify RNA sequence into sequences with or without pseudoknots. Combining neural network with grammar helps in reducing the drawbacks of both the technologies and increasing the overall power of identifying sequences with pseudoknots. Thus Learning Grammar is a better method of classifying pseudoknots.

\section{REFERENCES}

[1] Yuki Kato, Hiroyuki Seki, Tadao Kasami. RNA Pseudoknotted Structure Prediction Using Stochastic Multiple Context Free Grammar, IPSJ Digital Courier, 2006, 2:655-664.

[2] Rabindra Ku. Jena, Musbah M. Aqel, Pankaj Srivastava, Prabhat K. Mahanti. Soft Computing Methodologies in Bioinformatics, European Journal of Scientific Research, 2009, 26(2):189-203.

[3] B. A. Deiman, C. W. Pleij. Pseudoknots: A Vital Feature In Viral RNA, Seminars in Virology, 1997, 8(3):166-175.

[4] E. A. Rodland. Pseudoknots in RNA Secondary Structures: Representation, Enumeration, and Prevalence, Journal of Computational Biology, 2006, 13(6):11971213.

[5] Campbell, Mitchell, Reece. Biology Concepts and Connections, Second Edition, Addison Wesley, 1997
[6] D. W. Staple, S. E. Butcher, Pseudoknots: RNA Structures with Diverse Functions, PLoS Biology, 2005, 3(6).

[7] Thomas K.F. Wong, Y.S. Chiu, T.W. Lam, S.M. Yiu. Memory Efficient Algorithms For Structural Alignment of RNAs With Pseudoknots, IEEE Transactions On Computational Biology And Bioinformatics, 2007.

[8] R. M. Dirks and N. A. Pierce. A Partition Function Algorithm For Nucleic Acid Secondary Structure Including Pseudoknots, Journal of Computational Chemistry, 2003, 24(13):1664-1677.

[9] C.W.A. Pleij, K. Rietveld, L. Bosch. A New Principle of RNA Folding Based On Pseudoknotting, Nucleic Acids Research, 1985, 13(5):1717-1731.

[10] E. Rivas, S. Eddy. A Dynamic Programming Algorithm for RNA Structure Prediction Including Pseudoknots, Journal of Molecular Biology, 1999, 2053-2068.

[11] Han B, Dost B, Bafna V, Zhang S. Structural Alignment of Pseudoknotted RNA, Journal of Computational Biology, 2008, 15(5):489-504.

[12] Herbert H. Tsang, Kay C.. Wiese. SARNA-Predict-PK: Predicting RNA Secondary Structures Including Pseudoknots, IEEE, 2008.

[13] Liming Cai, L. Russell Malmberg, Yunzhou Wu. Stochastic Modeling of RNA Pseudoknotted Structure A Gramatical Approach, Bioinformatics, 2003, 19:66-73.

[14] Ankita Jiwan, Shailendra Singh. A Review on RNA Pseudoknot Structure Prediction Techniques, International Conference on Computing, Electronics and Electrical Technologies [ICCEET], IEEE sponsored, 2012, 975-978.

[15] Nobuyoshi Mizoguchi, Yuki Kato, Hiroyuki Seki. A Grammar-Based Approach to RNA Pseudoknotted Structure Prediction, IEEE, 2011, 135-140.

[16] Q. Liu, X. Ye, Y. Zhang. A Hopfield Neural Network Based Algorithm For RNA Secondary Structure Prediction, Proc. of the First International MultiSymposiums on Computer and Computational Sciences (IMSCCS'06), 2006, 1-7.

[17] Anne Condon, Beth Davy, Baharak Rastegari, Shelly Zhao, Finbarr Tarrant. Classifying RNA Pseudoknotted Structures, Theoretical Computer Science, 2004, 320(1) $: 35-50$

[18] Rafael García. Prediction of RNA Pseudoknotted Secondary Structure using Stochastic Context Free Grammars (SCFG), CLEI Electronic Journal, 2006.

[19] Shubhra Sankar Ray, Munia Bachhar, Sankar K. Pall. RNA Secondary Structure Prediction in Soft Computing Framework: A Review, IEEE, 2010.

[20] Denise R Koessler, Debra J Knisley, Jeff Knisley, Teresa Haynes. A Predictive Model For Secondary RNA Structure Using Graph Theory And A Neural Network, BMC Bioinformatics, 2010

[21] Cathy H. Wu. Artificial Neural Networks for Molecular Sequence Analysis, Computers Chem., 1997, 21(4):231256.

[22] Yang Liu Maozu Guo Quan Zou, Tuo Zhao. Predicting RNA Secondary Structure Based on The Class Information And Hopfield Network, Computers in Biology and Medicine, 2009, 39(3):206-214. 
[23] Yasuo Uemura, Aki Hasegawa, Satoshi Kobayashi, Takashi Yokomori. Tree Adjoining Grammars For RNA Structure Prediction, Theoretical Computer Science, 1999.

[24] Yuki Kato, Hiroyuki Seki, Tadao Kasami. Subclasses of Tree Adjoining Grammar for RNA Secondary Structure, Seventh International Workshop on Tree Adjoining Grammar and Related Formalisms, 2004.

[25] Timo Koskela, Mikko Lehtokangas, Jukka Saarinen, Kimmo Kaski. Time Series Prediction with Multilayer Perceptron, FIR and Elman Neural Networks, Proceedings of the 1996 World Congress on Neural Networks by International Neural Network Society

[26] B. de Vries, J. Principe The gamma model: A new neural net model for temporal processing, Neural Networks, 1992, 5:565-576.
[27] S. Haykin. Neural networks: A comprehensive foundation, Macmillan College Publishing Company, New York, 1994.

[28] Sergio Roa, Fernando Nino. Classification of Natural Language Sentences using Neural Networks, FLAIRS, 2003.

[29] J. Elman. Distributed Representations. Simple Recurrent Networks, and Grammatical Structure, Machine Learning, 1991.

[30] S. Haykin. Neural Networks: A Comprehensive Foundation, Upper Saddle River, N.J.: Prentice Hall, 1999.

[31] F. Pereira, Y. Shabes. Inside-Outside Re- Estimation from Partially Bracketed Corpora. In Proceedings of the 30th Annual Meeting of the Association for Computational Linguistics, 1992. 